\title{
Study of the Electromagnetic Waves Propagation over the Improved Fractal Sea Surface Based on Parabolic Equation Method
}

\author{
Wenwan Ding, ${ }^{1}$ Kun Wang, ${ }^{2}$ and Yunliang Long ${ }^{1}$ \\ ${ }^{1}$ Department of Electronics and Communication Engineering, Sun Yat-sen University, Guangzhou 510006, China \\ ${ }^{2}$ School of Information Engineering, Guangdong University of Technology, Guangzhou 510006, China \\ Correspondence should be addressed to Yunliang Long; isslyl@mail.sysu.edu.cn
}

Received 4 July 2016; Revised 18 September 2016; Accepted 19 October 2016

Academic Editor: Jaume Anguera

Copyright (C) 2016 Wenwan Ding et al. This is an open access article distributed under the Creative Commons Attribution License, which permits unrestricted use, distribution, and reproduction in any medium, provided the original work is properly cited.

An improved fractal sea surface model, which can describe the capillary waves very well, is introduced to simulate the onedimension rough sea surface. In this model, the propagation of electromagnetic waves (EWs) is computed by the parabolic equation (PE) method using the finite-difference (FD) algorithm. The numerical simulation results of the introduced model are compared with those of the Miller-Brown model and the Elfouhaily spectrum inversion model. It has been shown that the effects of the fine structure of the sea surface on the EWs propagation in the introduced model are more apparent than those in the other two models.

\section{Introduction}

Modeling the EWs propagation in marine environment is of great significance in the fields of the wireless communication, radar detection, GPS, and so on. In marine environment, to accurately simulate the actual sea surface is an essential task. At present, the existing sea surface models universally applied in electromagnetic computing include the MillerBrown model $[1,2]$, the spectrum inversion model, and the fractal model [3]. The Miller-Brown sea surface model is commonly used to simulate the EWs propagation on the sea surface [4]. The spectrum inversion method is applied to study the electromagnetic scattering on the sea surface at the first [5]. Then Benhmammouch applies the Elfouhaily spectrum [6] inversion model to study the influence of the rough sea surface on the EWs propagation in maritime environment $[7,8]$. Up to now, just the two models have been used to compute the EWs propagation. However, the MillerBrown model does not take the influence of the geometric sea surface on the EWs propagation into account. Though the Elfouhaily spectrum inversion model can show the influence of the structure of the sea surface on the EWs propagation, it cannot simulate the capillary waves finely. And furthermore, the expression of the Elfouhaily spectrum is miscellaneous. In fact, the fractal sea surface model can depict the sea surface finely and is also widely applied in electromagnetic computing $[9,10]$. Besides, the fractal geometry has been useful in antenna engineering to design small and multiband antennas as well [11]. Later, the improved fractal sea surface model $[12,13]$ is proposed. But this type of model is mainly used to study the electromagnetic scattering. So in order to simulate the fine structure of the sea surface efficiently and study the influence of the fine structure on the EWs propagation, the improved fractal sea surface model (onedimension) is introduced to study the EW propagation. Besides, the FD based PE method $[14,15]$ is applied to simulate the propagation of the EWs in this model because it is good at dealing with the complicated boundary.

This paper is mainly divided into three parts. In Section 2, the PE method and the finite-difference algorithm are described. In Section 3, the three models of the sea surface are discussed. The sea surface profiles depicted by the improved fractal model are shown and compared with those obtained by the Elfouhaily spectrum inversion model. In Section 4 , the EWs propagation losses in the three models are computed and compared, which have demonstrated the validity of the 


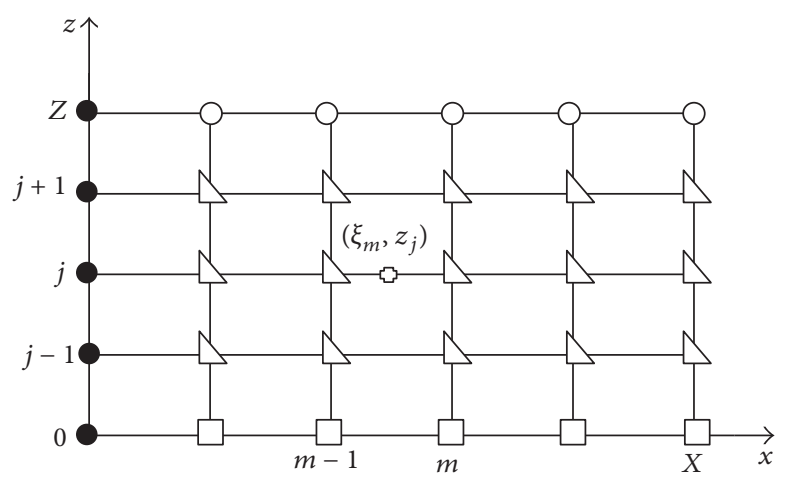

FIGURE 1: Finite-difference grid model.

introduced model and its ability of reflecting the effect of the capillary waves of the sea surface on the EWs propagation.

\section{Computing Method for Electromagnetic Wave Propagation}

2.1. Parabolic Equation and Finite-Difference Method. PE is derived from the wave equation under certain assumptions [14]. The equation itself automatically includes the effects of the refraction and diffraction of the EWs. It is a full wave analysis method. The expression of the parabolic equation is [15]

$$
\left(\frac{\partial}{\partial x}+i k(1-Q)\right) u=0
$$

where

$$
\begin{aligned}
& Q=\sqrt{\frac{1}{k^{2}} \frac{\partial^{2}}{\partial z^{2}}+n^{2}(x, z)}=\sqrt{1+Z} \\
& Z=\frac{1}{k^{2}} \frac{\partial^{2}}{\partial z^{2}}+n^{2}(x, z)-1,
\end{aligned}
$$

where $k$ is the EW wave number and $n$ is the refractive index. By applying the Padé approximation, (1) results in the socalled Claerbout equation with the form

$$
\begin{aligned}
& \frac{\partial^{3} u}{\partial x \partial z^{2}}-2 i k \frac{\partial^{2} u}{\partial z^{2}}+k^{2}\left(n^{2}+3\right) \frac{\partial u}{\partial x}-2 i k^{3}\left(n^{2}-1\right) u \\
& \quad=0
\end{aligned}
$$

The finite-difference method is adopted to solve the parabolic equation. In order to implement the equidistant rectangular finite difference, the region of the electromagnetic propagation field points is established as shown in Figure 1. The relation between the field points is shown by (4). The detailed solving process of the Claerbout equation can be found in [15].

$$
\begin{aligned}
\xi_{m} & =\frac{1}{2}\left(x_{m-1}+x_{m}\right) \\
u\left(\xi_{m}, z_{j}\right) & =\frac{1}{2}\left[u\left(x_{m-1}, z_{j}\right)+u\left(x_{m}, z_{j}\right)\right] .
\end{aligned}
$$

2.2. Boundary Condition. In order to solve the parabolic equation, the boundary condition should be considered properly. Here, we adopt the Leontovich impedance boundary condition expressed as [16]

$$
\begin{aligned}
\left.\frac{\partial u}{\partial z}\right|_{z=0}+\left.\beta u\right|_{z=0} & =0 \\
\beta & =i k \sin \theta\left(\frac{1-\Gamma}{1+\Gamma}\right),
\end{aligned}
$$

where $\Gamma$ is the Fresnel reflection coefficient and $\theta$ is the grazing angle.

2.3. Refractive Index. The refraction index $n$ is usually used to describe the information of the atmospheric environment. In this paper, the modified refractive index of Battaglia [17] is applied. The relation between the refraction index $n(z)$ and the modified refractive index $M(z)$ is defined as [18]

$$
n(z)=1-\frac{z}{R}+M(z) \times 10^{-6} .
$$

\section{Simulation of Sea Surface}

3.1. Miller-Brown Sea Surface Model. In the Miller-Brown sea surface model, the sea surface is approximated into a flat one. The influence of the rough sea surface on the propagation is reflected by the effective reflection coefficient $\Gamma_{e}[1,2]$; the expression is

$$
\Gamma_{e}=\rho \Gamma_{0},
$$

where $\Gamma_{0}$ is the reflection coefficient for a flat sea surface and $\rho$ is the roughness reduction factor, which is expressed as

$$
\rho=\int_{-\infty}^{\infty} \exp (2 i k \varsigma \sin \theta) P(\varsigma) d \varsigma,
$$

where

$$
P(\varsigma)=\frac{1}{\pi^{3 / 2} h} \exp \left(-\frac{\varsigma^{2}}{8 h^{2}}\right) K_{0}\left(\frac{\varsigma^{2}}{8 h^{2}}\right),
$$

where $K_{0}$ is the modified Bessel function of the second kind of order 0 and $h$ is the random mean square (rms) deviation of the sea surface height. 


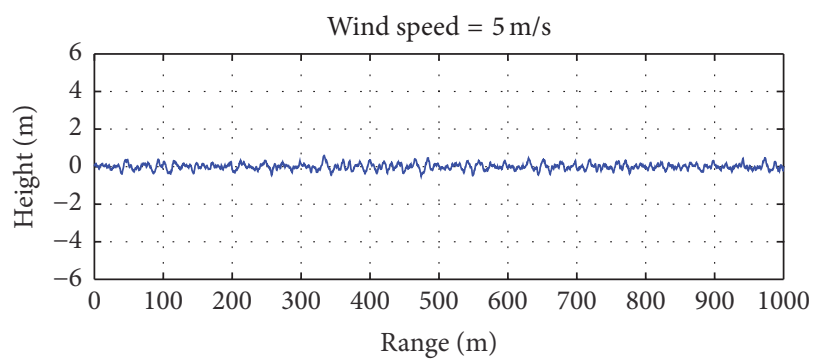

(a)

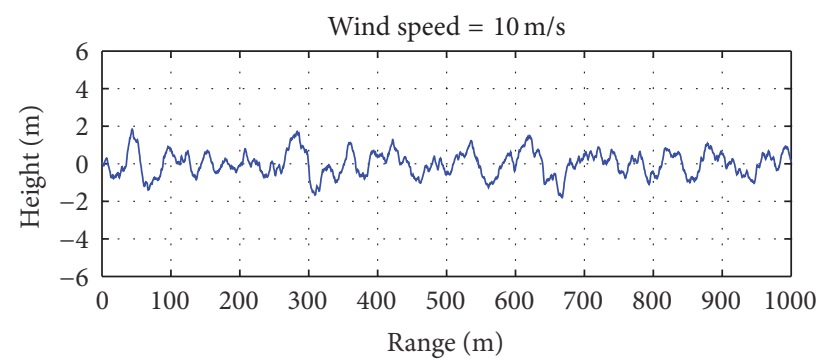

(b)

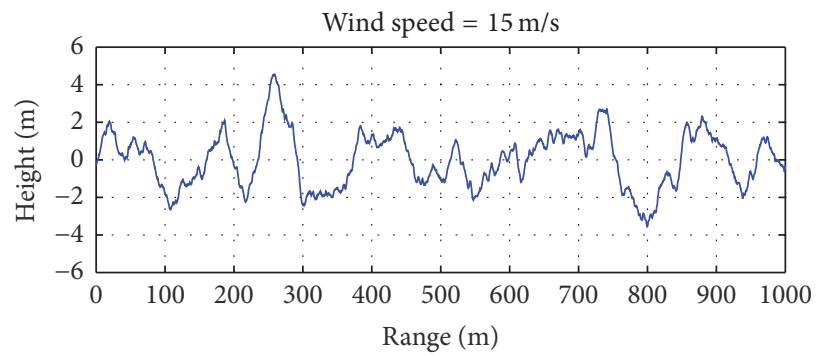

(c)

Figure 2: The sea surface profiles generated by the Elfouhaily spectrum inversion method with the wind speed: (a) $5 \mathrm{~m} / \mathrm{s}$, (b) $10 \mathrm{~m} / \mathrm{s}$, and (c) $15 \mathrm{~m} / \mathrm{s}$.

3.2. Elfouhaily Spectrum Inversion Sea Surface Model. The Elfouhaily spectrum is an omnidirectional and winddependent spectrum, and it is first proposed by Elfouhaily. This spectrum is valid over all wavenumbers and is amenable to electromagnetic models, and it is expressed as a sum of two spectrum regimes (long-wave curvature spectrum $B_{l}$ and short-wave curvature spectrum $B_{h}$ ) [6]:

$$
S(k)=k^{-3}\left(B_{l}+B_{h}\right) .
$$

With regard to the Elfouhaily spectrum, the sea surface is generated by the Monte-Carlo method. The introduction of the Monte-Carlo method can be seen in [19]. The sea surface profiles with different wind speeds are shown in Figure 2.

3.3. Improved Fractal Sea Surface Model. Since the fractal model can depict the structure of the sea surface more finely, it has been widely used in simulating the electromagnetic scattering on the sea surface $[9,10]$. But the power spectrum of the typical fractal model can only satisfy the negative power law index spectrum and cannot indicate the entire spectrum of the sea surface. So we adopt the following one-dimensional improved fractal model to simulate the sea surface $[12,13]$, and the improved model satisfies the global PM spectrum. The function of the sea surface height is defined as

$$
\begin{aligned}
& f(x, t) \\
& =\sigma \eta \sum_{m=0}^{M-1} a^{-(D-\xi) m} \cos \left[k_{0} a^{m}\left(x+v_{x} t\right)+\omega_{m} t+\beta_{m}\right] \\
& \quad+\sigma \eta \sum_{n=0}^{N-1} b^{(D-2) n} \cos \left[k_{0} b^{n}\left(x+v_{x} t\right)+\omega_{n} t+\beta_{n}\right],
\end{aligned}
$$

where $t$ is the time, $v_{x}$ is the speed of the receiving platform (the receiving platform is supposed to be immobile here, so $v_{x}$ is set to 0$), \eta$ is the normalized factor, $D$ is the fractal dimension, $a$ and $b$ are the scale factors, $\omega_{m}$ and $\omega_{n}$ are the angular frequencies of the waves, and $\beta_{m}$ and $\beta_{n}$ are the uniformly distributed random phases in $[-\pi, \pi]$. The wind speed at 19.5 meters above the sea surface $\left(U_{19.5}\right)$, the square root of sea surface height $(\sigma)$, and the fundamental wave number $\left(k_{0}\right)$ of the sea surface satisfy the following relation $[13,20]$ :

$$
\begin{gathered}
\sigma=\frac{0.0212 \zeta U_{19.5}}{4} \\
k_{0}=\frac{\omega_{0}^{2}}{g}=\frac{0.877^{2} g}{U_{19.5}^{2}},
\end{gathered}
$$

where $\xi=2.9, \zeta=1.65, b=1.015, a=1 / b, g=9.81 \mathrm{~m} / \mathrm{s}^{2}$, $D=1.62$, and $M=N=400$.

According to the above expressions, the sea surface profiles with different wind speeds are simulated, as shown in Figure 3. From Figures 2 and 3, we can see that the structure of the sea surface generated by the improved fractal method is more fine.

\section{The Propagation Results and Discussion}

In this paper, the Gaussian source [14] is applied, in which beam-width is $3^{\circ}$, the elevation angle is $0^{\circ}$, and the antenna (horizontal polarization) is at height $25 \mathrm{~m}$ above the surface. Now combining the contents of Sections 2 and 3, the EW propagation model is built, as shown in Figure 4, and the staircase terrain approximation method is used to deal with 


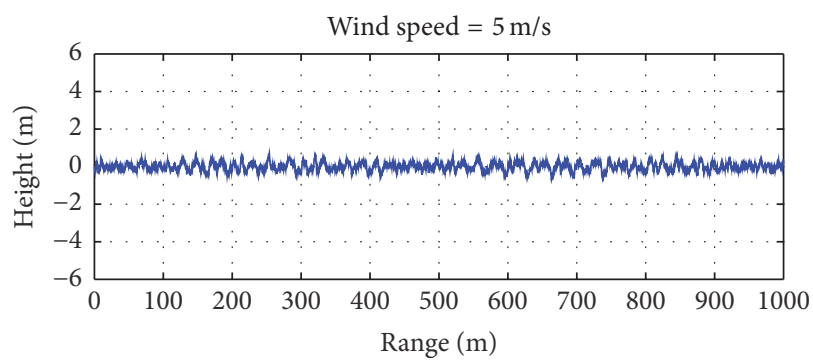

(a)

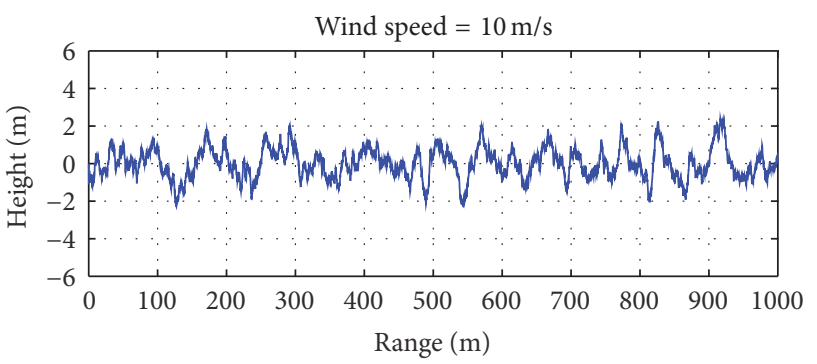

(b)

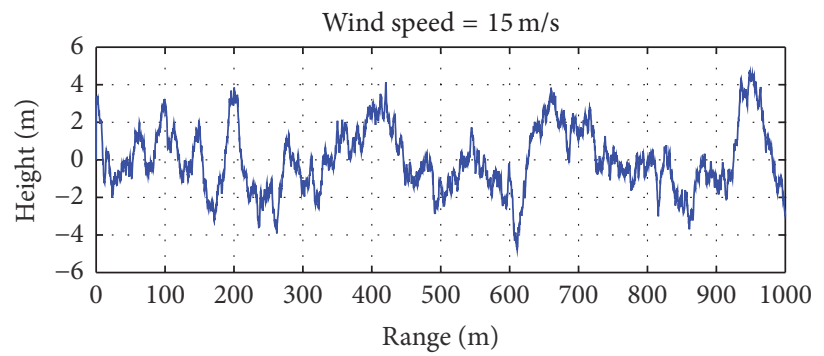

(c)

Figure 3: The sea surface profiles generated by the improved fractal method with the wind speed: (a) $5 \mathrm{~m} / \mathrm{s}$, (b) $10 \mathrm{~m} / \mathrm{s}$, and (c) $15 \mathrm{~m} / \mathrm{s}$.

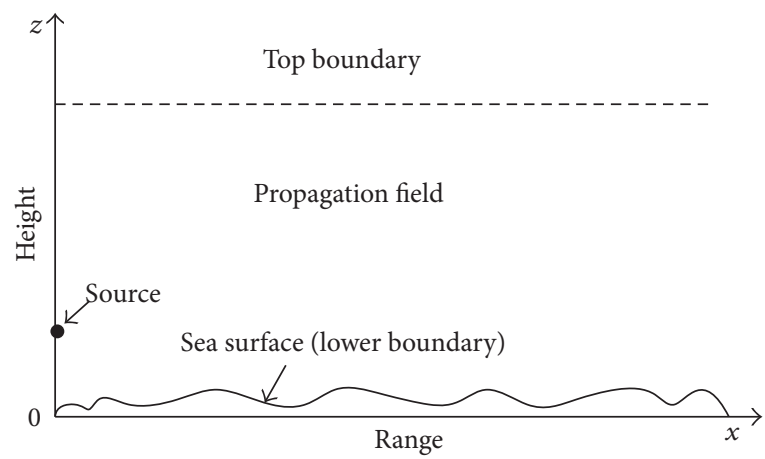

FIGURE 4: The EWs propagation model on the sea surface.

the irregular sea surface. According to the fineness of the sea surface structure in the improved fractal model, in the case of the mesh length longer than one meter, the finer meshing in the FDM and the finer structures of the sea surface will be taken into consideration. But when the mesh length is shorter than one meter, the mesh length will have little influence on the fineness of the sea surface in the propagation model. So considering the simulation efficiency, the mesh length of the FDM is set to double wavelengths of the EW in this paper.

Here the propagation loss (PL) is computed to represent the EWs propagation characteristics. The PLs in the improved fractal propagation model versus range $(0-5 \mathrm{~km})$ and height $(0-100 \mathrm{~m})$ with wind speed $\left(U_{19.5}\right) 5 \mathrm{~m} / \mathrm{s}, 10 \mathrm{~m} / \mathrm{s}$, and $15 \mathrm{~m} / \mathrm{s}$ are computed and shown in Figure 5. As shown in Figure 3, the height of the sea wave increases with the wind speed, and from Figure 5, we notice that the influence of the rough sea surface on the EWs propagation increases with the wind speed.
In order to show the effects of the fine structure of the sea surface on the EWs propagation, we study this issue mainly from two aspects. First, the effect of the rough sea surface with different wind speeds on the EWs propagation is researched. The PLs at a distance of $3000 \mathrm{~m}$ with different wind speeds are simulated and shown in Figure 6. For comparison, the height of the sea surface in the Miller-Brown model is set to be the same as that of the other two models (which is got from experiments). As shown in Figure 6, the fluctuation of the PL curves of the three models is almost consistent when the wind speed is low $(5 \mathrm{~m} / \mathrm{s}$, e.g., Figure $6(\mathrm{a}))$. When the wind speed increases (from $5 \mathrm{~m} / \mathrm{s}$ to $15 \mathrm{~m} / \mathrm{s}$ ), the deviation of the PL curves in the three models also increases. Therefore, on the one hand, it demonstrates the validity of the improved fractal sea surface propagation model. On the other hand, it indicates that the structure of the rough sea surface has a great influence on the propagation. In addition, we can also notice that the change rates of the PL at the height in the improved 


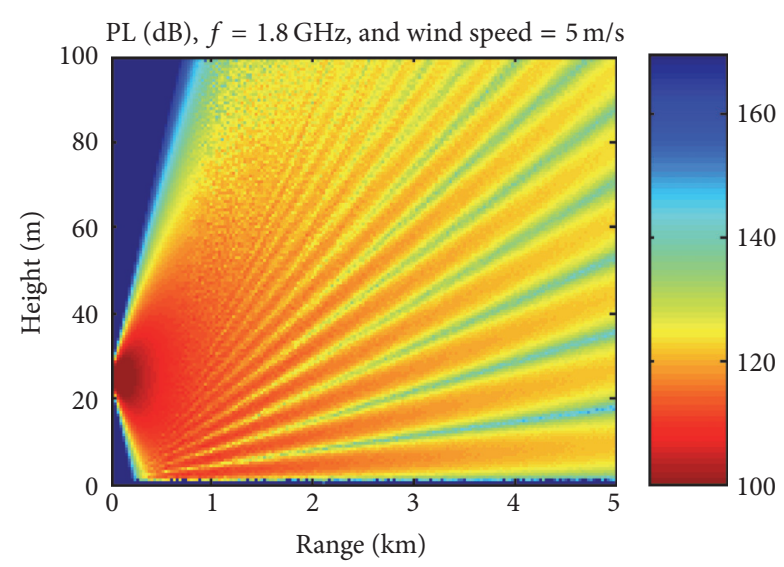

(a)

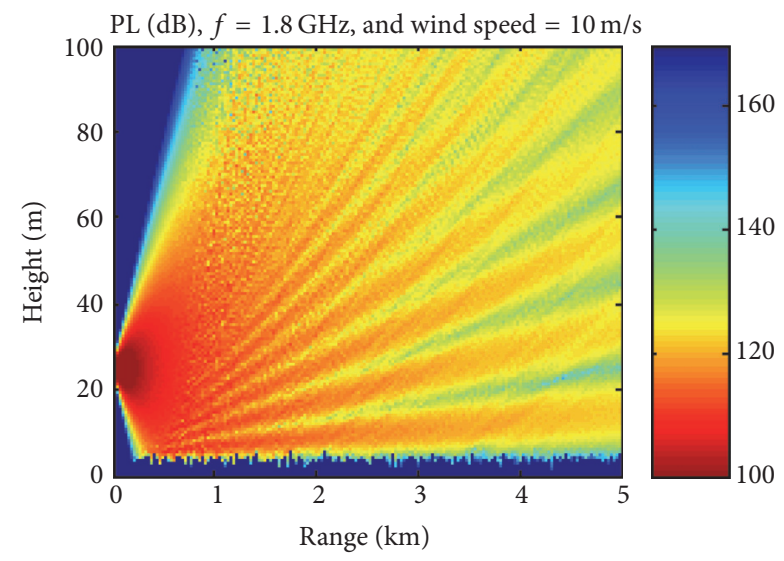

(b)

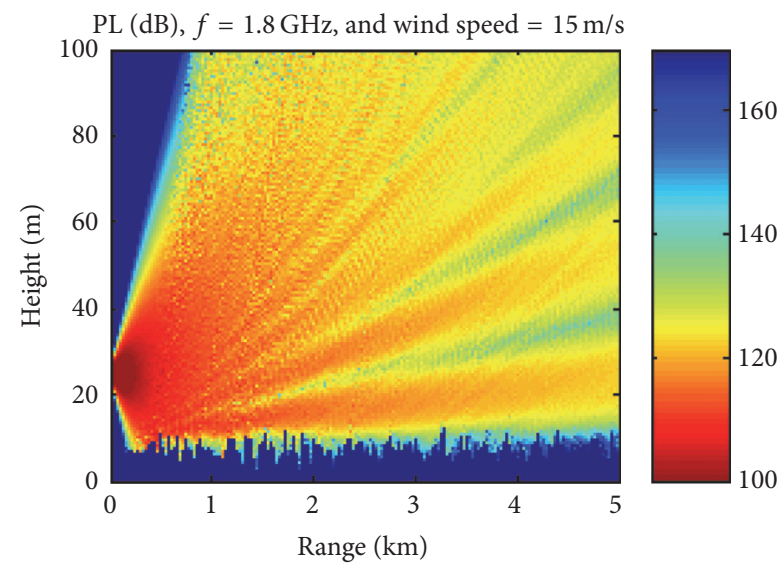

(c)

Figure 5: Propagation in the improved fractal model with the wind speed: (a) $5 \mathrm{~m} / \mathrm{s}$. (b) $10 \mathrm{~m} / \mathrm{s}$, and (c) $15 \mathrm{~m} / \mathrm{s}$.

fractal propagation model are much faster than those in the other two models, and the variation scope of the PL in the improved fractal model is greater than that in the other two models at the same height. This is because more details of the sea surface in this model are depicted. So in theory, the improved fractal sea surface model is closer to the realistic sea surface.

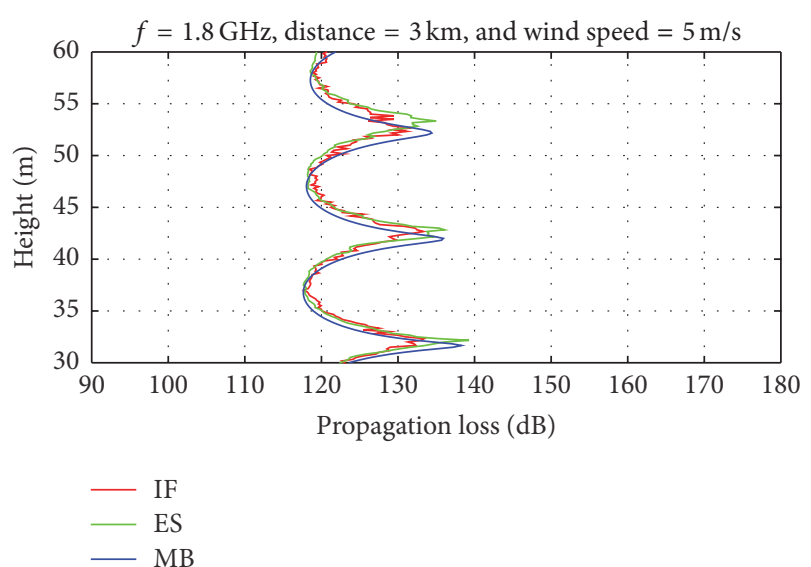

(a)

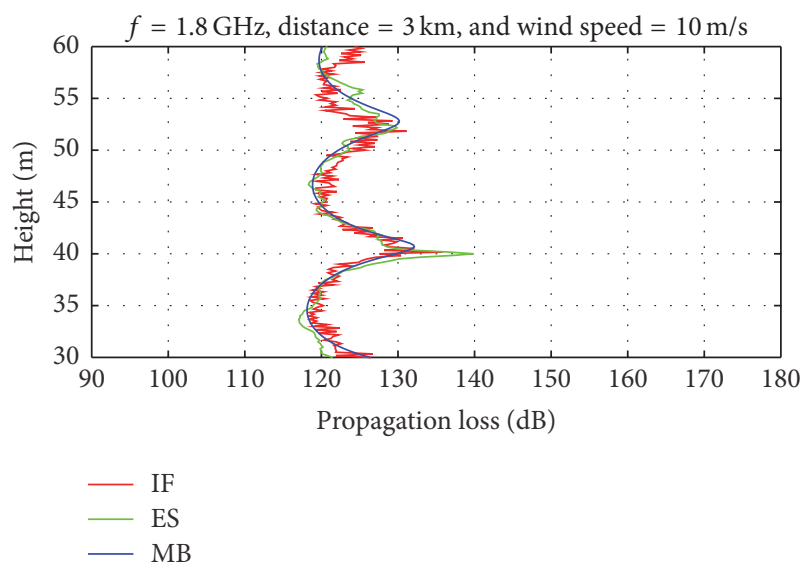

(b)

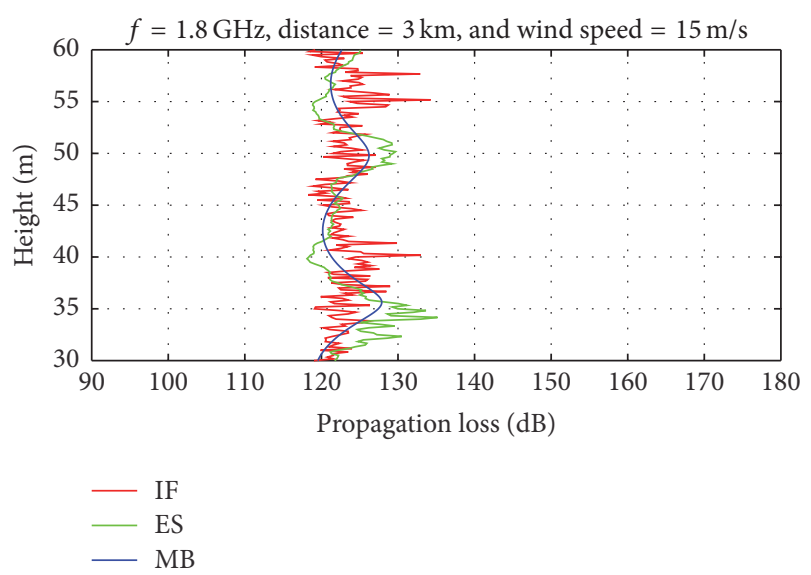

(c)

FIGURE 6: PLs in the three propagation models at a horizontal distance of $3000 \mathrm{~m}$ with the wind speeds: (a) $5 \mathrm{~m} / \mathrm{s}$, (b) $10 \mathrm{~m} / \mathrm{s}$, and (c) $15 \mathrm{~m} / \mathrm{s}$.

Then, the effect of the fine structure of the sea surface on the different frequencies EWs propagation is studied. The frequencies of the EWs in UHF of IEEE $(0.5 \mathrm{GHz})$, Lband $(1.5 \mathrm{GHz}), \mathrm{S}$-band $(3 \mathrm{GHz})$, and C-band $(6 \mathrm{GHz})$ are used to study the propagation in the sea surface models. The PLs of different frequencies EWs propagation at a distance 


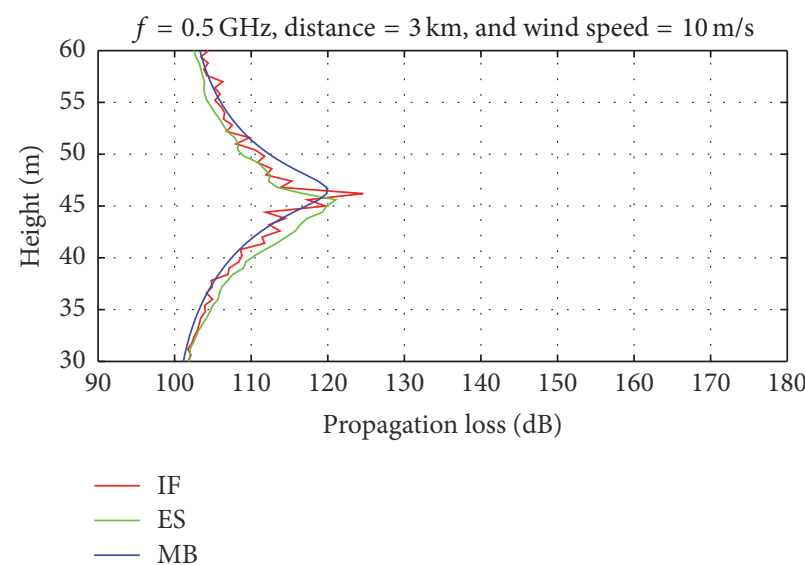

(a)

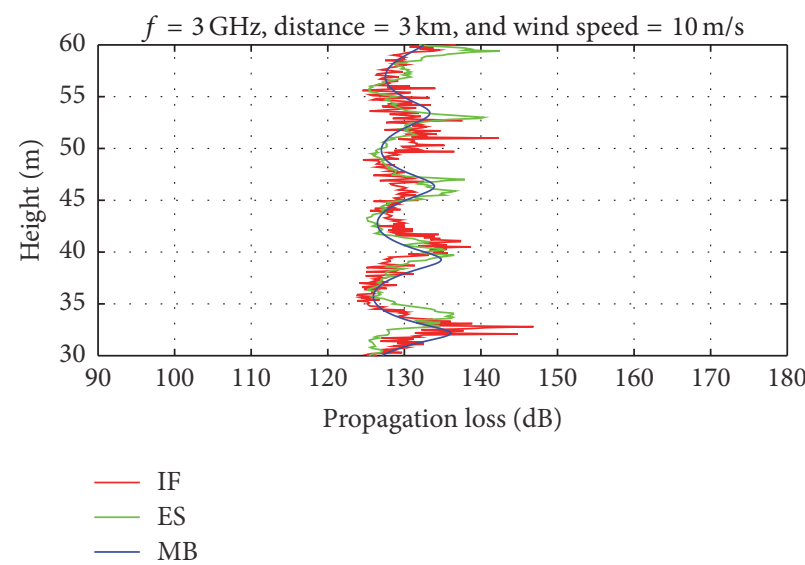

(c)

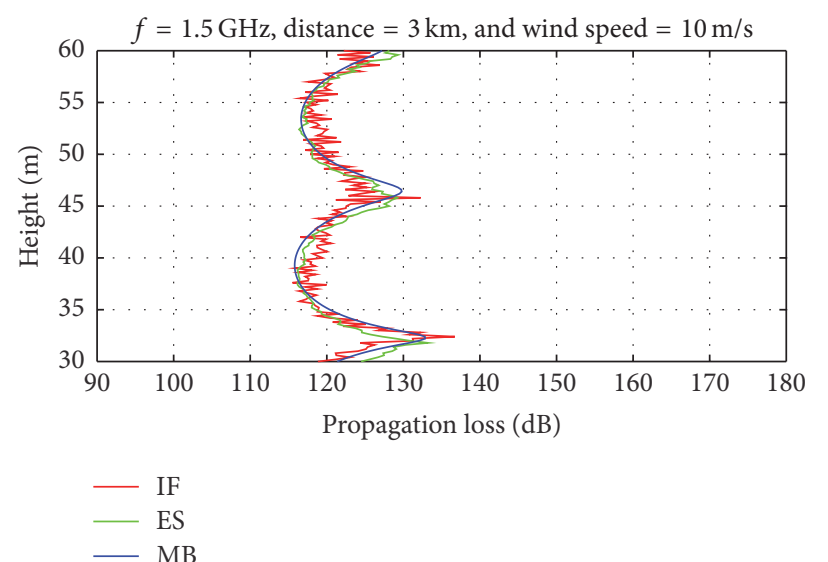

(b)

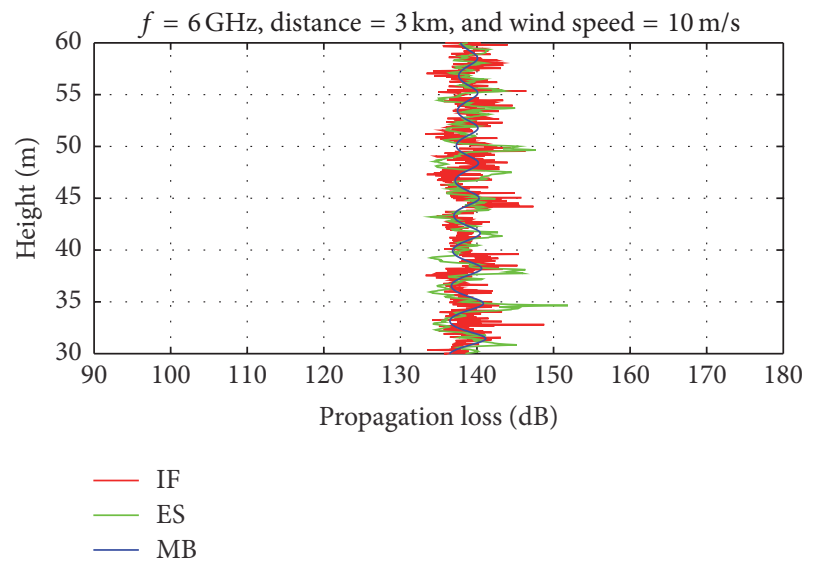

(d)

FIGURE 7: PLs in the three propagation models at a horizontal distance of $3000 \mathrm{~m}$, when the frequencies of the EWs are (a) $0.5 \mathrm{GHz}$, (b) $1.5 \mathrm{GHz}$, (c) $3 \mathrm{GHz}$, and (d) $6 \mathrm{GHz}$.

of $3000 \mathrm{~m}$ with the same wind speed $(10 \mathrm{~m} / \mathrm{s})$ in the three models are simulated and shown in Figure 7. From Figure 7, it is not difficult to see that the influence of the sea surface structure on the propagation of high frequency EWs is greater than that of the low frequency EWs, and the effects of the improved fractal sea surface model on the PLs are greater than that of the other two models. When the frequency of the EWs increases to $6 \mathrm{GHz}$, the effect of the fine structure of the sea surface on the PL is great and it should not be ignored. So in this case, using the Miller-Brown model and the Elfouhaily spectrum inversion model to simulate the EWs propagation on the sea surface will cause a relatively large error, especially the Miller-Brown model.

In fact, when the wind speed increases, the deviations between the three sea surface models and the realistic sea surface also increase. This is because the foams are generated on the realistic sea surface, but we do not take it into consideration in our models. In addition, according to the wind scale, the wave rolls will appear when the wind speed is higher than $20 \mathrm{~m} / \mathrm{s}$. Under this situation, all of the three models are not suitable for simulating the sea surface anymore and it should be simulated by the more matched models, such as the Fournier model [21].

\section{Conclusions}

By computing the EWs propagation losses in the three models, it is clear that the fine structure of the sea surface has a significant influence on the propagation, and the influence increases with the wind speed and the frequency of the EWs. From the comparison between the improved fractal model and the Elfouhaily spectrum inversion model, we can find that there is more detailed information of the sea surface in the improved fractal sea surface model. It indicates that the simulation of the EWs propagation in the improved fractal sea surface model is more close to the actual situation on the sea surface. Besides, in the process of simulating, we find that the expressions of the improved fractal model are simpler than the Elfouhaily spectrum inversion model, which make it easier to establish model. Therefore, in theory, the introduced model in this paper is more suitable to be applied to study the EWs propagation over the sea surface.

Furthermore, the one-dimension improved fractal sea surface model could also be extended to the two-dimension model, which can be applied to predict the 3D EWs propagation. In addition, this sea surface model could be combined with the other models (such as the Fournier model) to simulate the sea surface under different conditions. 


\section{Competing Interests}

The authors declare no competing interests regarding the publication of this paper.

\section{Acknowledgments}

This work was supported by the NSF of China (41376041 and 61401106), the NSF of Guangdong Province (2015A030312010 and 2014B090901057), and Research Fund for the Doctoral Program of Higher Education of China (20130171110024).

\section{References}

[1] A. R. Miller, R. M. Brown, and E. Vegh, "New derivation for the rough-surface reflection coefficient and for the distribution of sea-wave elevations," IEE Proceedings H: Microwaves Optics and Antennas, vol. 131, no. 2, pp. 114-116, 1984.

[2] M. Levy, "Propagation over the rough sea surface," in Parabolic Equation Methods for Electromagnetic Wave Propagation, chapter 10, section 6, IEE Press, London, UK, 2000.

[3] F. Berizzi, E. Dalle Mese, and G. Pinelli, "One-dimensional fractal model of the sea surface," IEE Proceedings-Radar, Sonar and Navigation, vol. 146, no. 1, pp. 55-63, 1999.

[4] D. E. Freund, N. E. Woods, H.-C. Ku, and R. S. Awadallah, "Forward radar propagation over a rough sea surface: a numerical assessment of the Miller-Brown approximation using a horizontally polarized 3-GHz line source," IEEE Transactions on Antennas and Propagation, vol. 54, no. 4, pp. 1292-1304, 2006.

[5] J. V. Toporkov and G. S. Brown, "Numerical simulations of scattering from time-varying, randomly rough surfaces," IEEE Transactions on Geoscience and Remote Sensing, vol. 38, no. 4, pp. 1616-1625, 2000.

[6] T. Elfouhaily, B. Chapron, K. Katsaros, and D. Vandemark, "A unified directional spectrum for long and short wind-driven waves," Journal of Geophysical Research C: Oceans, vol. 102, no. 7, pp. 15781-15796, 1997.

[7] O. Benhmammouch, A. Khenchaf, and N. Caouren, "Modelling roughness effects on propagation of electromagnetic waves in a maritime environment: a hybrid approach," IET Radar, Sonar and Navigation, vol. 5, no. 9, pp. 1018-1025, 2011.

[8] O. Benhmammouch, A. Khenchaf, and N. Caouren, "Electromagnetic waves propagation above rough sea surface application to evaporation ducts," in Proceedings of the IEEE International Geosicence and Remote Sensing Symposium (IGARSS '08), pp. 1189-1192, Boston, Mass, USA, July 2008.

[9] T. Lo, H. Leung, J. Litva, and S. Haykin, "Fractal characterisation of sea-scattered signals and detection of sea-surface targets," IEE Proceedings, Part F: Radar and Signal Processing, vol. 140, no. 4, pp. 243-250, 1993.

[10] F. Berizzi and E. D. Mese, "Scattering coefficient evaluation from a two-dimensional sea fractal surface," IEEE Transactions on Antennas and Propagation, vol. 50, no. 4, pp. 426-434, 2002.

[11] J. Anguera, C. Puente, C. Borja, and J. Soler, "Fractal shaped antennas: a review," in Encyclopedia of RF and Microwave Engineering, John Wiley \& Sons, New York, NY, USA, 2005.

[12] Y.-H. Wang, L.-X. Guo, and Z.-S. Wu, "The application of an improved 1D fractal model for EM scattering from sea surface," Acta Electronica Sinica, vol. 35, no. 3, pp. 478-483, 2007 (Chinese).
[13] Y.-H. Wang, L.-X. Guo, and Z.-S. Wu, “The application of an improved 2D fractal model for electromagnetic scattering from the sea surface," Acta Physica Sinica, vol. 55, no. 10, pp. 5191-5199, 2006.

[14] M. Levy, "Parabolic equation algorithms," in Parabolic Equation Methods for Electromagnetic Wave Propagation, chapter 3, section 6, pp. 35-41, IEE Press, London, UK, 2000.

[15] P. D. Holm, "Wide-angle shift-map PE for a piecewise linear terrain-a finite-difference approach," IEEE Transactions on Antennas and Propagation, vol. 55, no. 10, pp. 2773-2789, 2007.

[16] A. Leontovitch, On the Approximate Boundary Conditions for an Electromagnetic Filed on the Surface of Well-conducting Bodies, Investigations of Propagation of Radio Waves, Academy of Science, Moscow, Russia, 1948.

[17] M. R. Battaglia, "Modelling the radar evaporative duct," RANL Technical Note 3/85, Department of Defence, Defence Science and Technology Organisation, Weapons Systems Research Laboratory, RAN Research Laboratory, Australia, 1985.

[18] A. E. Barrios, "Parabolic equation modeling in horizontally inhomogeneous environments," IEEE Transactions on Antennas and Propagation, vol. 40, no. 7, pp. 791-797, 1992.

[19] G. P. Bein, "Monte Carlo computer technique for onedimensional random media," IEEE Transactions on Antennas and Propagation, vol. 21, no. 1, pp. 83-88, 1973.

[20] G. P. Harrison and A. R. Wallace, "Climate sensitivity of marine energy," Renewable Energy, vol. 30, no. 12, pp. 1801-1817, 2005.

[21] A. Fournier and W. T. Reeves, "A simple model of ocean waves," ACM SIGGRAPH Computer Graphics, vol. 20, no. 4, pp. 75-84, 1986. 


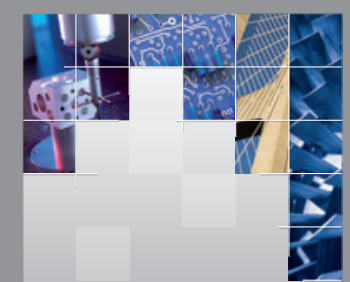

\section{Enfincering}
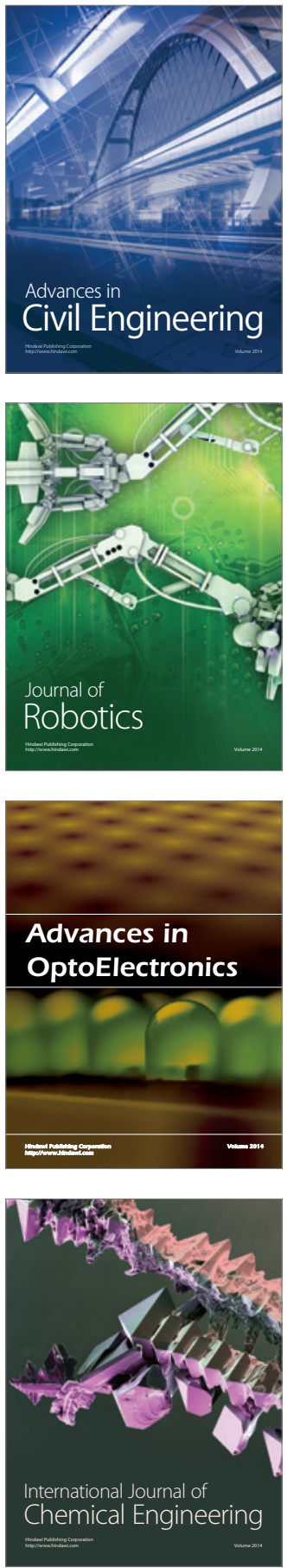

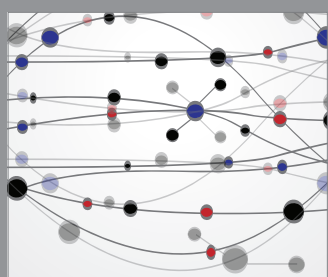

The Scientific World Journal

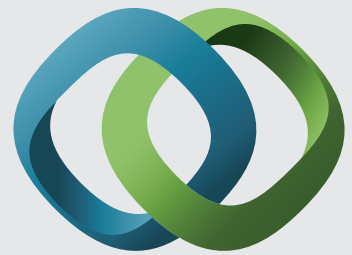

\section{Hindawi}

Submit your manuscripts at

http://www.hindawi.com
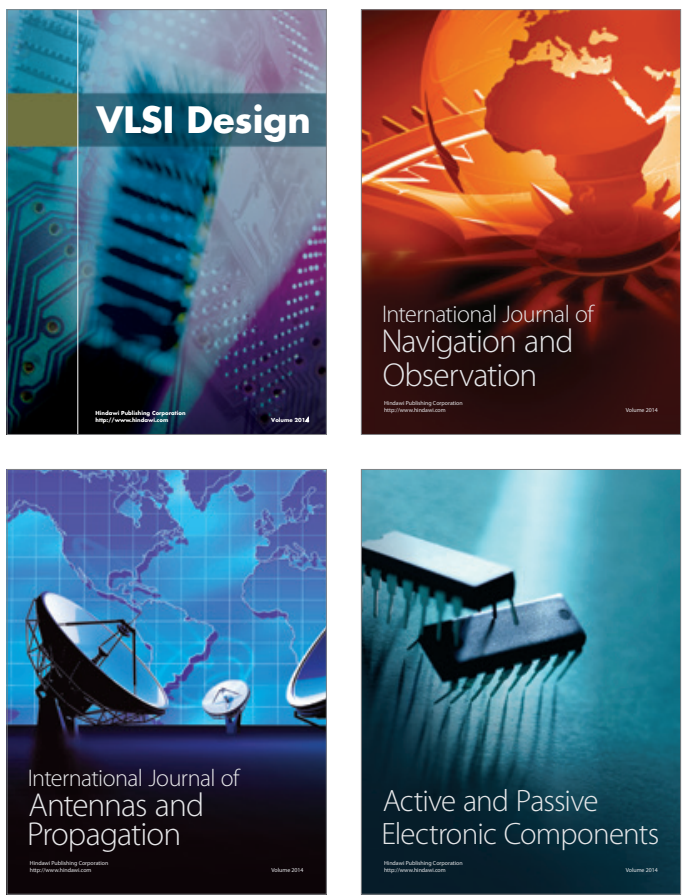
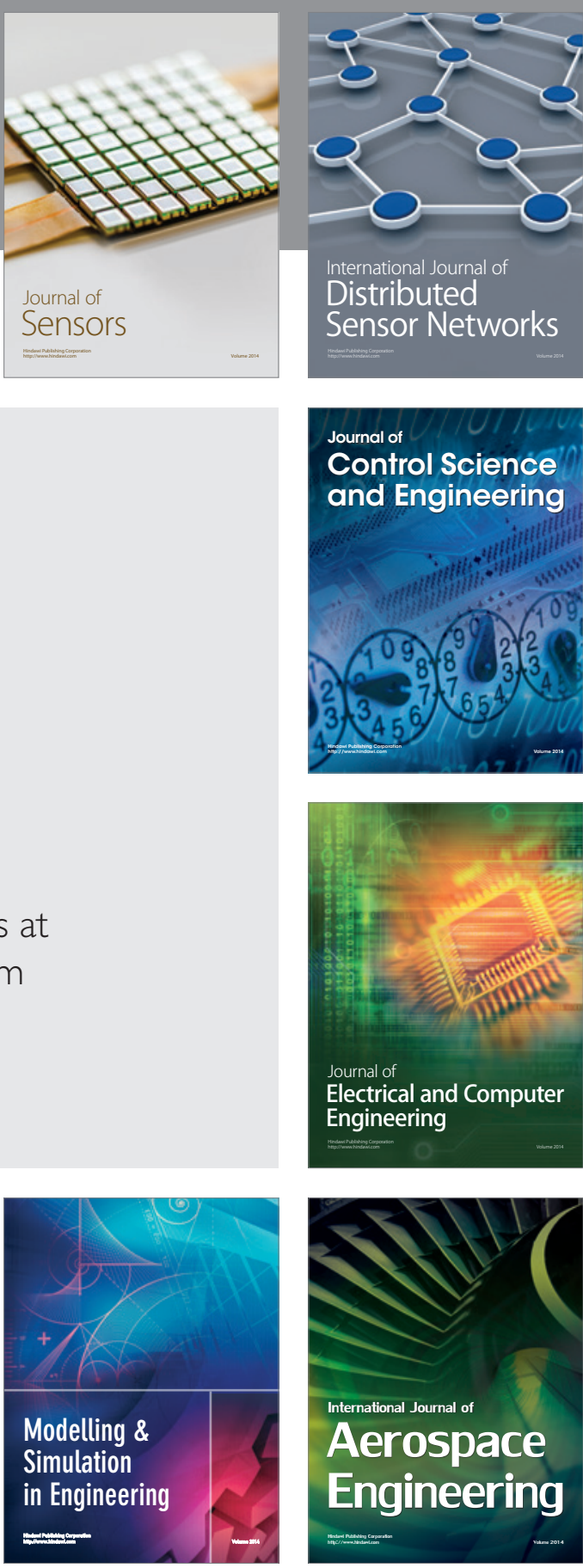

International Journal of

Distributed

Sensor Networks

Journal of

Control Science

and Engineering
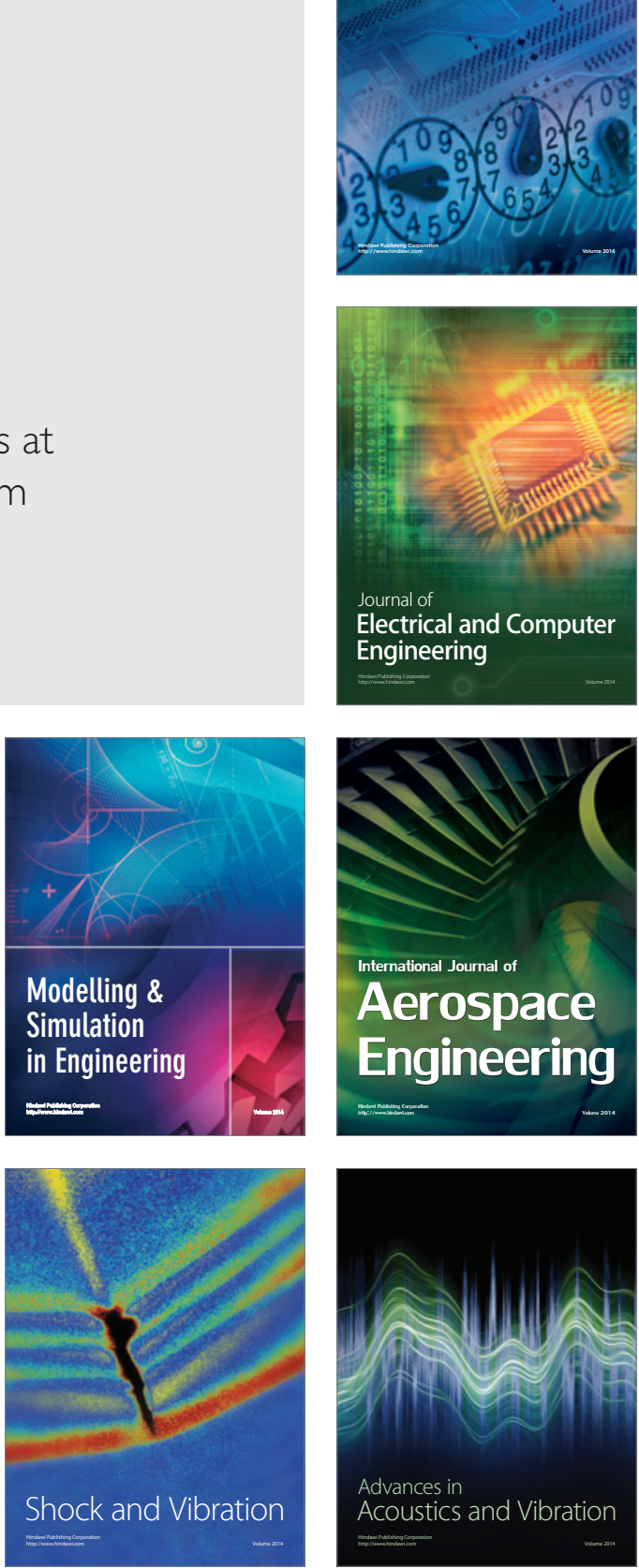